\title{
Femtosecond-laser Nanostructuring in Glass
}

\author{
Yasuhiko SHIMOTSUMA ${ }^{* 1}$, Taiga ASAI $^{1}$, Masaaki SAKAKURA ${ }^{2}$ and Kiyotaka MIURA ${ }^{1}$ \\ ${ }^{1}$ Department of Material Chemistry, Kyoto University, Kyotodaigaku-Katsura, Nishikyo-ku, Kyoto \\ 615-8510, Japan \\ E-mail: yshimo@func.mc.kyoto-u.ac.jp \\ ${ }^{2}$ Office of Society-Academia Collaboration for Innovation, Kyoto University, Yoshida-Honmachi, \\ Sakyo-ku, Kyoto, 606-8501, Japan
}

\begin{abstract}
We demonstrate the polarization-dependent anisotropy of the laser induced nanostructures inside $\mathrm{SiO}_{2}$ and $\mathrm{GeO}_{2}$ glass. Such nanostructures show form birefringence which is controllable by the femtosecond double-pulse configuration. In the case of $\mathrm{SiO}_{2}$ glass, we have also demonstrate polarization imaging filter based on the linear dichroism for the visible light ranging from 400 to $800 \mathrm{~nm}$. While, in the case of $\mathrm{GeO}_{2}$ glass, the decomposition of constituent elements according to increase in laser energy was also observed.
\end{abstract}

DOI: $10.2961 / \mathrm{jlmn} .2014 .01 .0007$

Keywords: $\mathrm{SiO}_{2}$ glass, $\mathrm{GeO}_{2}$ glass, femtosecond laser, nanostructure, anisotropy

\section{Introduction}

Material processing with ultrafast lasers has recently attracted considerable interest [1] due to a wide range of applications including laser surgery [2], 3D micro- and nanostructuring [3]. According to the relation between the laser interpulse time and the thermal diffusion in materials, thermal accumulation effect can be observed around the focus, followed by the element distribution derived from the temperature gradient [4]. An intriguing phenomenon which currently attracts a lot of interest is self-assembly of periodic nanostructures in the direction perpendicular to the light polarization [3]. The observations suggest that, in a certain intensity range, the interference between longitudinal electron plasma waves leads to the formation of nanosized gratings with a pitch as small as $150 \mathrm{~nm}$ [3]. These periodic nanostructures are ruled in the direction parallel to the polarization of the writing laser and consist of thin regions with a low refractive index characterized by a strong oxygen deficiency [3], surrounded by larger regions with a positive index change [5]. Uniaxial birefringence observed after femtosecond laser irradiation of $\mathrm{SiO}_{2}$ glass [6] has been explained by induced nanogratings and referred as self-assembled form birefringence [7]. More recently, Bhardwaj et al. proposed the nanoplasmonic model for the formation of the nanoplanes generated from local field enhancements caused by the inhomogeneous breakdown [8]. They proposed that the underdense nanoplasmas grow perpendicularly to the polarization into sheets, which is similar to the light propagation in planar metallic waveguides. While the predicted grating period of $\Lambda=\lambda / 2 n$ is agreement with experimental results, the refractive index change is not fully understood [9]. Such a periodic assembly behaves as a uniaxial negative birefringent material which is consistent with a planar form birefringence, whose optical axis is parallel to the direction of the polarization of the writing laser. It has been shown that these self-assembled nanostructures indicate the local refractive index change $(\sim$ -0.1 ) with respect to the unprocessed material, change due to variation of the pulse duration [10]. More recently, nanogratings are shown to consist of mesoporous nanoplanes, assuming that the nanopore formation is due to glass decomposition leading to the generation of molecular oxygen [11]. In the case of $\mathrm{SiO}_{2}$ glass, self-assembled nanostructure has initially evolved from residual birefringence originated from internal stress distribution. Evolution of optical anisotropy in glass can be controlled as a function of interpulse time due to thermal accumulation. Such phenomenon is particularly prominent for the longer interpulse interval corresponding to the lower thermal accumulation effect, in spite of the same pulse energy [12]. Various applications ranging from embedded micro-reflectors [13], retardation plates [5], micro-fluidic channels [10], to rewritable 5D optical storage [7] based on these nanostructures have been reported; however the mechanism including dynamics of self-organized nanostructures formation is still not fully understood. Here, by using $\mathrm{SiO}_{2}$ glass, we report that control of slow axis orientation and phase retardation of form birefringence induced by femtosecond double pulse configuration. We also demonstrate a polarization imaging filter. We also discuss the formation of periodic nanostructure inside $\mathrm{GeO}_{2}$ glass. The decomposition of constituent elements of $\mathrm{GeO}_{2}$ glass, which includes generation of molecular oxygen $[14,15]$, can be also observed according to increase in laser energy.

2. Control of form birefringence in $\mathrm{SiO}_{2}$ glass using temporally shaped femtosecond double pulse

The experiments were performed using a mode-locked, regeneratively amplified Ti: Sapphire laser system (Coherent; RegA 9000), operating at $800 \mathrm{~nm}$ with $70 \mathrm{fs}$ pulse duration and $250 \mathrm{kHz}$ repetition rate. To understand the interaction between material and temporally shaped femtosecond laser pulse, the linearly polarized double pulses were produced from the reshaping of amplified pulses by using acoustic optic phase dispersion filter (AOPDF, Fastlite Inc.; Dazzler). The double-pulse train with various time delays $\tau_{\text {delay }}$ (Fig. 1(a)) and intensity ratios $I^{1 \text { st }} / I^{\text {tot }}$ between double pulses were obtained by tuning the second-order dispersion 
component (Fig. 1(b)). In this experiments, the interpulse time, $\tau_{\text {int }}$ was set to $0.1 \mathrm{~ms}$. The double-pulse train was focused inside a fused silica a microscope objective (Nikon; LU Plan Fluor, $50 \times 0.80$ N.A.) at a depth of about 100 $\mu \mathrm{m}$ below the sample surface. The pulse energy of double pulses was about $0.68 \mu \mathrm{J}$ and the beam power measured after microscope objective was independent on the orientation of light polarization. The modified regions are inspected by a polarization microscope (CRi Inc.; LC-Polscope) for evaluation of the induced phase retardation.
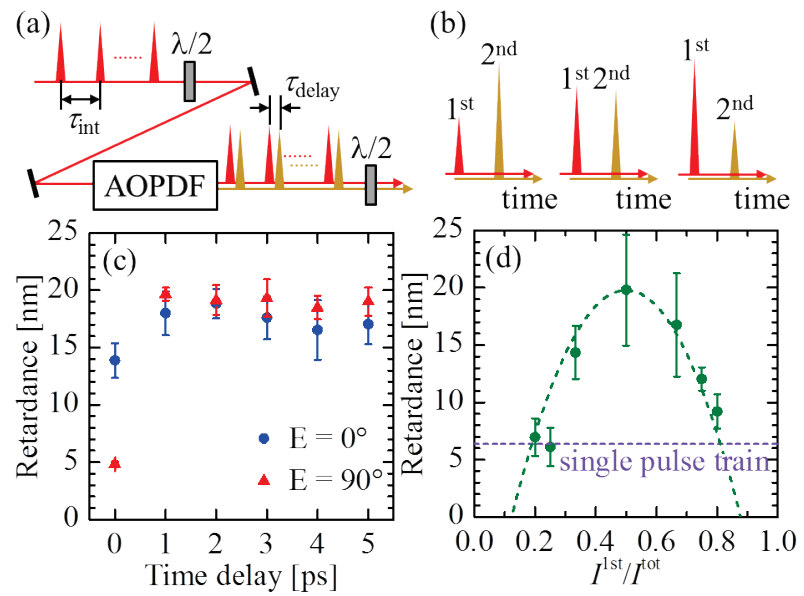

Fig. 1 (a) Schematic of temporally shaped double pulse configuration: acoustic optic phase dispersion filter (AOPDF), half-wave plate $(\lambda / 2) . \tau_{\text {int }}$ and $\tau_{\text {delay }}$ indicate an interpulse time and a time delay between double pulses, respectively. (b) Pulse diagrams of linearly polarized double pulses with various intensity ratios. Plots of the phase retardance versus (c) $\tau_{\text {delay }}$ between double pulses and (d) intensity ratios of the first-arriving pulse to the total double pulse intensity $I^{\text {st }} / I^{\text {tot }}\left(I^{\text {tot }}=I^{1 \mathrm{st}}+I^{\text {ndd }}\right)$ at $\tau_{\text {delay }}=3 \mathrm{ps}$. Dashed purple and green lines in (d) indicate the retardance for the single-pulse train and a quadratic fitting for eye guidance, respectively.

The phase retardance induced by the double-pulse train was larger than that of the single-pulse train, regardless of polarization direction. Unexpectedly, no apparent difference in phase retardance can be observed in different time delay $\left(\tau_{\text {delay }}\right)$ between double pulses (Fig. 1(c)). On the other hand, it was found that there is a difference in the effect on retardance value induced by the linearly polarized double pulse with a different polarization (Fig. 1(c)). This phenomenon could be interpreted in terms of the enhancement of generation of free electron via the laser-plasma interaction of inverse bremsstrahlung during relaxation time of plasma excited by the first arriving pulse [16]. Interestingly, the stress accumulation depends on the polarization direction in the case of the single-pulse train, which could be explained by the anisotropy of electron plasma absorption for $\mathrm{p}$ and $\mathrm{s}$ polarizations at the oblique interface produced by the pulse with tilted intensity front. Heating anisotropy in homogeneous medium could be also originated form the correlation between a light polarization and a pulse front tilt [7]. Such anisotropy affects the reciprocal evolution of the material modification in isotropic medium, finally exhibits a non-reciprocal quill writing effect [17]. In order to control the effect of interaction between double-pulse train and plasma, the intensity ratios between the first arriving pulse $\left(I^{1 \mathrm{st}}\right)$ and the total intensity of double pulses $\left(I^{\text {tot }}=I^{\text {st }}\right.$
$+I^{\text {nd }}$ ) were also varied (Fig. 1(d)). In the experiments, $\tau_{\text {delay }}$ and $I^{\text {tot }}$ of double-pulse train was fixed at 3 ps and $0.68 \mu \mathrm{J}$, respectively. The maximum phase retardance was induced by the one-to-one double-pulse configuration. Symmetric variation of the induced phase retardance to the intensity ratio $\left(I^{1 \mathrm{st}} / I^{\mathrm{tot}}\right)$ could be as following. The energy first-arriving pulse is transferred to the glass through multiphoton absorption followed by the formation of electronhole pairs (multiphoton ionization). Once the free electron has become non-zero, further absorption leads the increase of temperature (due to electron absorption) and electron plasma density (due to avalanche). Such electron plasma firstly relaxes by electron-phonon coupling, and then radiative and/or non-radiative annihilation, followed transformation into point defects (e.g. $\mathrm{SiE}^{\prime}$ and $\mathrm{NBOHC}$ ), finally into an oxygen deficient center (ODC(II)). When the second pulse arrives before the electron plasma induced by the first-arriving pulse completely decays, the second pulse is employed to assist avalanche ionization [18]. Due to a difference in the dominant electron excitation process, for example, multiphoton ionization by the first-arriving pulse and avalanche ionization by the second arriving-pulse, symmetric variation of the induced phase retardance to the one-to-one double-pulse train could be observed. Further investigations will be required to fully understand the interaction between material and temporally shaped femtosecond laser pulse.

\section{Application to real-time polarization imaging filter}

Based on the measurements of the Mueller matrix, the modified region indicates the linear dichroism for the visible light ranging from 400 to $800 \mathrm{~nm}$ [19]. In order to estimate the modification in silica glass induced by femtosecond irradiation, we observed emission and excitation spectra of femtosecond-laser modified regions in a synthetic fused silica (Shin-Etsu Quartz; VIOSIL-SQ), containing approximately $500 \mathrm{ppm} \mathrm{OH}$. The measurements have been performed at room temperature using the spectrofluorometer (Horiba Jobin Yvon; Fluoro Max-3).

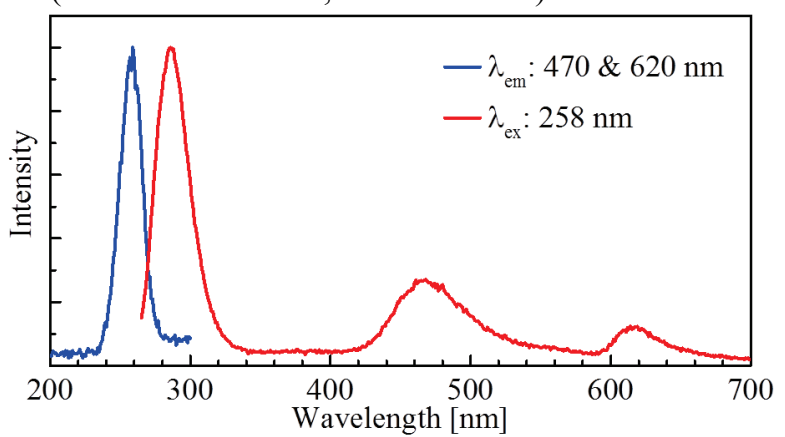

Fig. 2 Emission excited at $258 \mathrm{~nm}$ (red line) and excitation monitoring emission at 470 and $620 \mathrm{~nm}$ (blue line) spectra of femtosecond-laser modified regions.

Fig. 2 indicates emission and excitation spectra of femtosecond-laser modified regions. It should be noted that no apparent emission was observed in the initial glass. The peaks at about 283 and $470 \mathrm{~nm}$ can be assigned to ODC(II) [20]. Furthermore, the emission peak of $620 \mathrm{~nm}$ which is attributed to non-bridging oxygen hole center (NBOHC) was also observed [21]. More recently, Poumellec et al. have shown that the luminescence related to silicon oxygen 
deficient center (SiODC) is much stronger when the excitation polarization is parallel to the sample scanning direction and moved at low velocity, regardless of the writing polarization direction. This indicates that the creation of the anisotropic defects is oriented by the movement of the femtosecond laser beam [22]. We have demonstrated the polarization imaging CCD sensor monolithically implemented polarizer embedded in the $\mathrm{SiO}_{2}$ cover glass.

Polarization direction of incident light

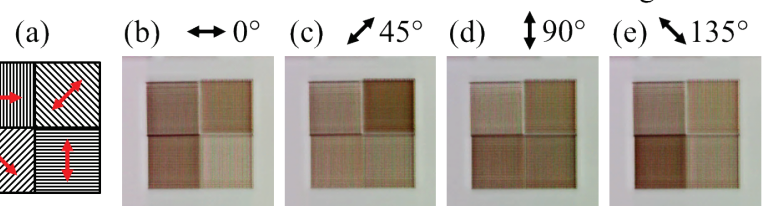

Fig. 3 (a) Diagram of the polarization imaging filter. Red arrows indicate the femtosecond-laser polarization direction which is perpendicular to the transmission axis of polarization imaging filter. Optical microscope images of the polarization filter array illuminated with (b) $0^{\circ}$, (c) $45^{\circ}$, (d) $90^{\circ}$ and (e) $135^{\circ}$ polarized incident light.

Owing to the multiple array of the polarizer configured with form birefringent nanostructures, the CCD pixels show polarization-dependent sensitivity (Fig. 3). The polarization filter array is composed of four distinct filters which are offset by 45 degrees. Fig. 3(b)-(e) show optical microscope images from the polarization filter array back illuminated with four different angles of polarized light. In $0^{\circ}$ and $90^{\circ}$ polarized light illumination (Fig. 3(b), (d)), the $0^{\circ}\left(90^{\circ}\right)$ filters are bright since the incident light is parallel to the transmission axis of the filters, while the $90^{\circ}\left(0^{\circ}\right)$ filters with a transmission axis perpendicular to the light polarization are dark. The attenuation of $45^{\circ}$ and $135^{\circ}$ filters appear in the same brightness and are slightly small compared to that of $0^{\circ}$ and $90^{\circ}$ filters. Fig. 3(c) and (e) also demonstrate the extinction behavior for the $45^{\circ}$ and $135^{\circ}$ polarized light illumination. The brightness of $0^{\circ}$ and $90^{\circ}$ filters is also slightly different from the $45^{\circ}$ and $135^{\circ}$ filters. Ideally, the attenuation of $0^{\circ}$ and $90^{\circ}$ filters under the $45^{\circ}$ and $135^{\circ}$ polarized incident light should be half of the incident light intensity. More detailed experiments are necessary to optimize the sensitivity of the polarization imaging filter.

\section{Polarization-dependent nanostructure in $\mathbf{G e O}_{2}$ glass}

The $\mathrm{GeO}_{2}$ glass sample was prepared by melting the starting powder at $1600{ }^{\circ} \mathrm{C}$ in a $\mathrm{Pt}$ crucible during $3 \mathrm{~h}$ and rapidly quenching it to room temperature. Femtosecond laser pulse operating at $800 \mathrm{~nm}$ with $70 \mathrm{fs}$ pulse with various energy $\left(E_{p}\right)$ from $80 \mathrm{~nJ}$ to $2.4 \mu \mathrm{J}$ was focused inside a $\mathrm{GeO}_{2}$ glass, via a microscope objective (Nikon; LU Plan Fluor, $100 \times 0.80$ N.A.). The pulse repetition rate and the irradiation time were set to $250 \mathrm{kHz}\left(\tau_{\text {int }}=4 \mu \mathrm{s}\right)$ and $1 \mathrm{~s}$, respectively. After laser irradiation, the modified structures were inspected using optical and polarization microscopes. A confocal Raman spectrometer (Tokyo Instruments; Nanofinder 30) was used for structural identification of the irradiated regions. Furthermore, to reveal nanostructure formation inside $\mathrm{GeO}_{2}$ glass, the sample surface was processed by FIB (JEOL; JIB-4600F) to the depth of the beam waist location. The surface of the FIB-processed sample was analyzed by scanning electron microscope (JEOL,
JSM-6700F). Fig. 4(a) indicates optical micrograph of the induced structures inside $\mathrm{GeO}_{2}$ glass by focused femtosecond laser pulses with various pulse energies $\left(\mathrm{E}_{\mathrm{p}}\right)$. The size of the modified region inside $\mathrm{GeO}_{2}$ glass dramatically changes with increasing $E_{p}$. Especially, when the $E_{p}$ is higher than $400 \mathrm{~nJ}$, the morphology of the induced structure has several circular structures due to the heat accumulation. The inner structure has been attributed to the photoexcitation and modification due to shock wave generation, on the other hand, the outer structure has been attributed to the temperature-dependent viscosity and the viscoelastic behavior under a stress loading by thermal expansion [23]. Even though it is expected to be stronger heat accumulation induced by higher pulse repetition rate, the size changes of the modified region in $\mathrm{SiO}_{2}$ glass with increasing number of pulses were independent of the interpulse interval [24]. This phenomenon could be caused by a difference between the thermal expansion coefficient of $\mathrm{SiO}_{2}\left(\sim 5 \times 10^{-7} \mathrm{~K}^{-1}\right)$ and $\mathrm{GeO}_{2}\left(\sim 8 \times 10^{-6} \mathrm{~K}^{-1}\right)$ [25]. Fig. 4(b) indicates the phase retardance as a function of pulse energy for $\mathrm{SiO}_{2}$ and $\mathrm{GeO}_{2}$ glass. These results suggest that polarization-dependent nanostructure was also induced inside $\mathrm{GeO}_{2}$ glass, additionally indicate that the threshold for nanostructure formation inside $\mathrm{GeO}_{2}$ glass is lower than that of $\mathrm{SiO}_{2}$ glass.

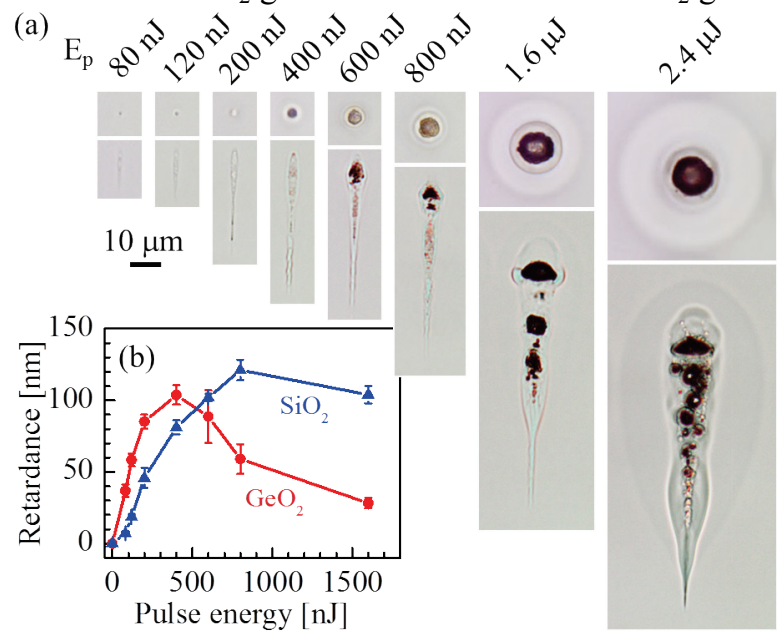

Fig. 4 (a) Characteristic micrographs of the induced structures inside $\mathrm{GeO}_{2}$ glass by focused femtosecond laser pulses with various pulse energy $\left(\mathrm{E}_{\mathrm{p}}\right)$. (b) Plots of the phase retardance versus pulse energy for $\mathrm{SiO}_{2}$ and $\mathrm{GeO}_{2}$ glass.
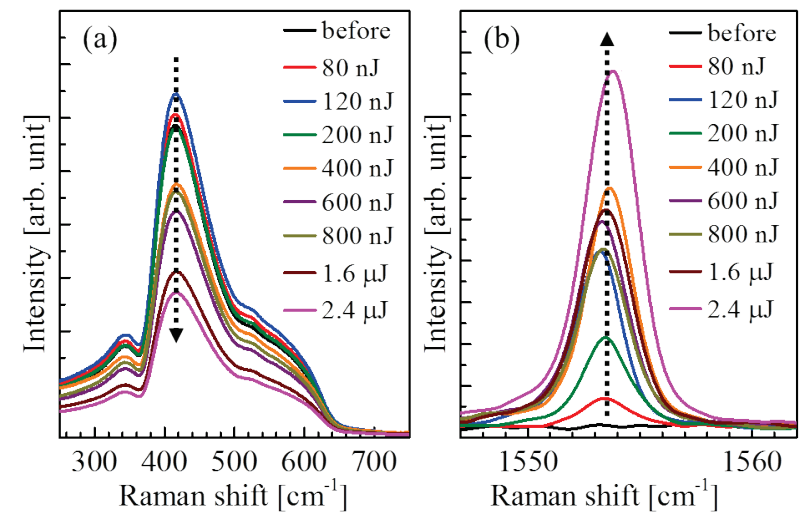

Fig. 5 Sequential Raman spectra taken as a function of laser energy from $80 \mathrm{~nJ}$ to $2.4 \mu \mathrm{J}$ at (a) low and (b) high wavenumber region. Raman spectrum of initial $\mathrm{GeO}_{2}$ glass is also shown. Dotted arrows show the observed tendencies with increasing pulse energy. 
The Raman spectra of the modified region, taken as a function of $E_{p}$, have been also observed (Fig. 5). To improve the data quality, each Raman spectra have been subtracted a luminescence background and corrected for temperature and frequency dependence of Raman scattering [26]. All the Raman bands have been assigned [27]. For example, the dominant band at $420 \mathrm{~cm}^{-1}$ has been attributed to the symmetric stretching of the $\mathrm{Ge}-\mathrm{O}-\mathrm{Ge}$ linkages. The $344 \mathrm{~cm}^{-1}$ band appears the motion of Ge (with little Omotion) [15]. The D2 band at $520 \mathrm{~cm}^{-1}$ has been attributed to the breathing of three-ring tetrahedral- $(\mathrm{GeO})_{4}$ structures. The broadband between $550 \sim 650 \mathrm{~cm}^{-1}$ is due to $\mathrm{Ge}-\mathrm{O}-\mathrm{Ge}$ bending motions. This band is important for obtaining the structural information in the structural modification inside $\mathrm{GeO}_{2}$ glass, because this band comes from the compaction of glass network structure. Indeed, Juodkazis et al. have also observed a decrease of the bands at $420 \mathrm{~cm}^{-1}$ and 344 $\mathrm{cm}^{-1}$, an increase of the D2 band $\left(520 \mathrm{~cm}^{-1}\right)$ after the femtosecond laser irradiation [15]. They have also observed a generation of molecular oxygen confined in a void inside $\mathrm{GeO}_{2}$ glass [14]. In our case, we have also observed a decrease of the bands at $420 \mathrm{~cm}^{-1}$ and $344 \mathrm{~cm}^{-1}$. Although the tendency of the D2 peak intensity according to laser energy is apparently different from the previous work, we have confirmed the decrease in the ratio of $520 \mathrm{~cm}^{-1}$ to $420 \mathrm{~cm}^{-1}$ (Fig. 5(a)). Additionally we have also observed molecular $\mathrm{O}_{2}$ peak at about $1550 \mathrm{~cm}^{-1}$, indicating $\mathrm{GeO}_{2}$ glass was decomposed into $\mathrm{Ge}$ and $\mathrm{O}$. The $\mathrm{O}_{2}$ peak intensity also increased with increasing in the pulse energy (Fig. 5(b)).

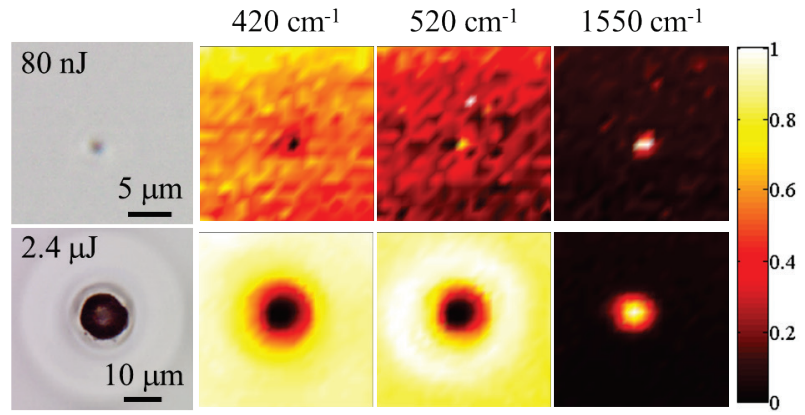

Fig. 6 Raman spectral maps of modified structures inside $\mathrm{GeO}_{2}$ glass by the femtosecond laser pulses with a different pulse energy of $80 \mathrm{~nJ}$ (upper low) or $2.4 \mu \mathrm{J}$ (lower row).

Fig. 6 shows the Raman spectral maps of modified structures scanned at the $420 \mathrm{~cm}^{-1}, 520 \mathrm{~cm}^{-1}$ and $1550 \mathrm{~cm}^{-1}$ band. In the case of low pulse energy $(80 \mathrm{~nJ})$, no apparent variation of the peaks at $420 \mathrm{~cm}^{-1}$ and $520 \mathrm{~cm}^{-1}$ were observed. In the central modified region, the peak at $1550 \mathrm{~cm}^{-}$ ${ }^{1}$ indicating the generation of molecular oxygen was slightly detected. On the other hand, in the case of high energy $(2.4 \mu \mathrm{J})$, a distinct molecular $\mathrm{O}_{2}$ peak was observed in the focal point. Furthermore, the peak intensity at $520 \mathrm{~cm}^{-1}$ decrease at the focal point and increased in a ring-shaped region around the focal point. These results suggest that the densification around the focus originated from the compressive stress by the thermal expansion of the central region [23]. It should be noted that the peak intensity at 420 $\mathrm{cm}^{-1}$ was decreased monotonically from circumference to center of focal point. To clarify the three-dimensional shape of the modified region with the high pulse energy, we have also observed Raman spectral maps of a cross-section structure (Fig. 7). Fig. 7 clearly indicates that the $\mathrm{O}_{2}$ was embedded inside the void structures in the center region. Furthermore, a slight decrease in intensity of the $420 \mathrm{~cm}^{-1}$ band and a slight increase in intensity of the defect band D2 $\left(520 \mathrm{~cm}^{-1}\right)$ around the center part including large voids could be associated with the densification and/or increase in fictive temperature [15].
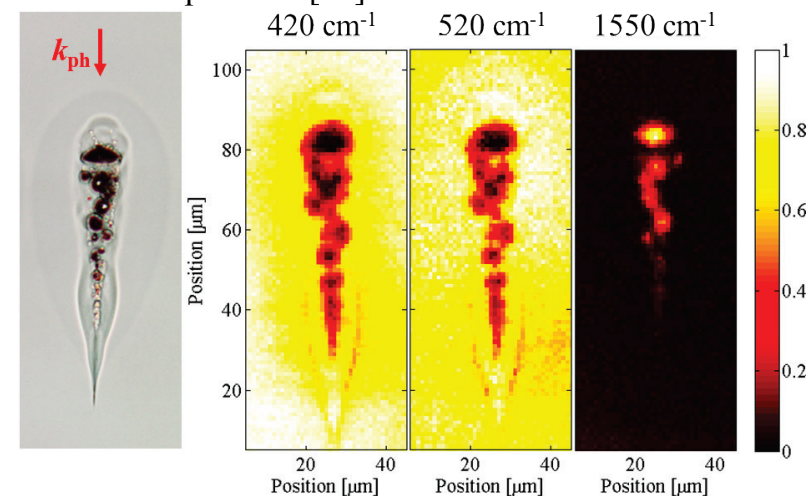

Fig. 7 Raman spectral maps of a cross-section structure inside $\mathrm{GeO}_{2}$ glass, induced by the focused femtosecond laser pulses of $2.4 \mu \mathrm{J}$. Arrow of $k_{\mathrm{ph}}$ indicates the incident light wave vector.

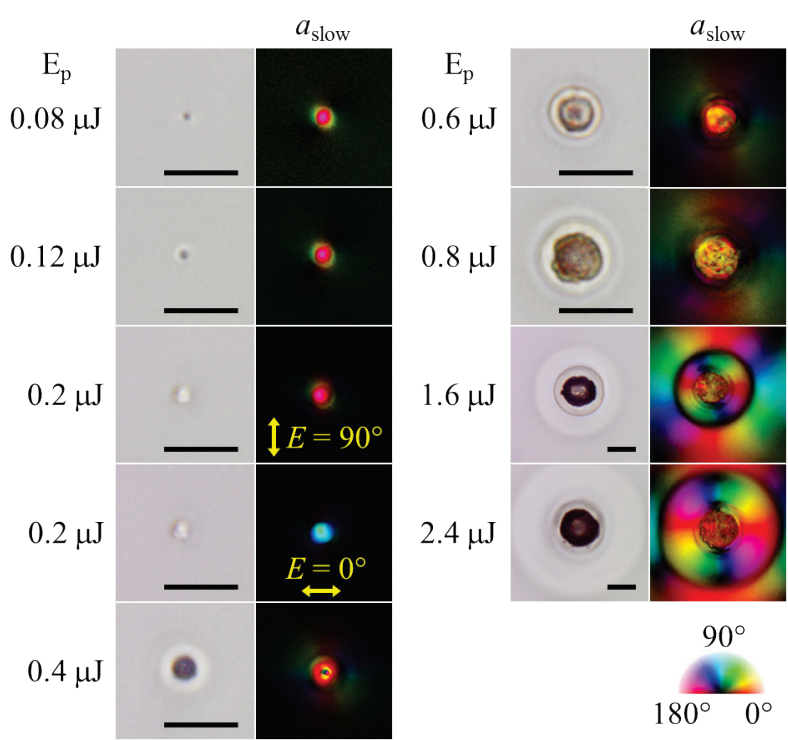

Fig. 8 Characteristic micrographs of the induced structure inside $\mathrm{GeO}_{2}$ glass by focused femtosecond laser pulses with various pulse energy $\left(\mathrm{E}_{\mathrm{p}}\right)$, taken with optical (left) and polarization (right) microscope (pseudo color indicates direction of the slow axis, $a_{\text {slow }}$, see polar legend). At the pulse energy of $0.2 \mu \mathrm{J}$, two different experiments by using the orthogonal polarized femtosecond laser pulses $\left(\mathrm{E}=0^{\circ}\right.$ or $\left.90^{\circ}\right)$ have been performed. Scale bars indicate $5 \mu \mathrm{m}$.

In order to reveal the origin of the birefringence in the modified region, we observed the birefringence with a polarization microscope (Fig. 8). The form birefringence can be observed within the focus area for the lower pulse energy than $0.2 \mu \mathrm{J}$. As is the case with $\mathrm{SiO}_{2}$ glass, the slow axis orientation is also perpendicular to the laser polarization (see $\mathrm{E}=0^{\circ}$ and $90^{\circ}$ at $0.2 \mu \mathrm{J}$ in Fig. 8), suggesting the nanograting structure could be self-organized inside $\mathrm{GeO}_{2}$ glass for lower pulse energy $(<0.2 \mu \mathrm{J})$. On the other hand, in the case of higher pulse energy $(>0.4 \mu \mathrm{J})$, the residual strain distribution was observed (in particular, $>0.6 \mu \mathrm{J}$ ). The slow axis orientation in the outer-modified region is 
distributed radially resulting from the compression by the thermal expansion in the central high-temperature region during laser irradiation.

To confirm the formation of form birefringent nanostructures in the case of lower pulse energy experiments, the $\mathrm{GeO}_{2}$ glass surface was processed by FIB (JEOL; JIB-4600F) to the depth of the laser beam waist location. Then the FIB-processed sample surface was analyzed by scanning electron microscope (JEOL, JSM6700F). Fig. 9 shows backscattering electron images (BEI) of the FIB-processed sample surface to the depth of focal spot location inside $\mathrm{GeO}_{2}$ glass. Since the BEI is sensitive to the atomic weight of the elements or the density of material constituting the observation surface, the BEI reveal a periodic structure of striped dark regions with low density of material and of $58 \mathrm{~nm}$ width which are also aligned perpendicular to the writing laser polarization direction. This phenomenon is the same as the nanograting structures formed inside $\mathrm{SiO}_{2}$ glass. The period of self-organized nanostructures was about $207 \mathrm{~nm}$. The oxygen defects could be also formed in the striped dark regions. In the same way as $\mathrm{SiO}_{2}$ glass, the striped dark regions could be also interpreted in terms of the formation of the oxygen defects created by the interaction between incident light field and the generated electron plasma.

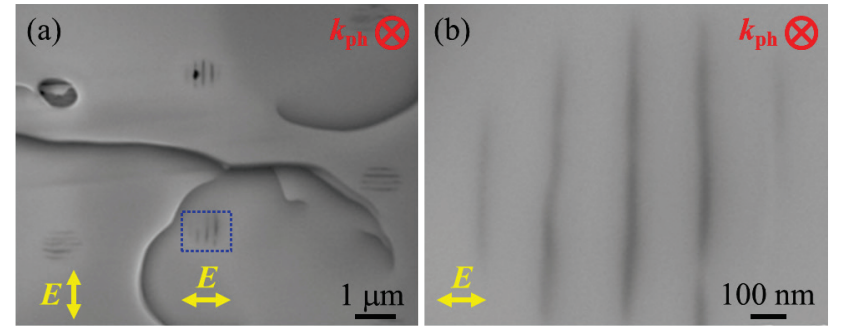

Fig. 9 Backscattering electron images of the FIB-processed sample surface to the depth of focal spot location inside $\mathrm{GeO}_{2}$ glass. The images are shown in two different scales. Low-magnification image (a) and high-magnification image (b) of boxed region in (a) are shown. $k_{\mathrm{ph}}$ and $E$ indicate the wave vector and the polarization direction of the incidence, respectively. The nanograting structures induced by the femtosecond laser pulses with two different polarization directions are also shown in (a).

\section{Conclusion}

In summary, the research using the temporally shaped femtosecond pulses indicates that the induced phase retardance is variable. Symmetric variation of the induced phase retardance to the one-to-one double-pulse train could be interpreted in terms of the individual electron excitation processes, for example, multiphoton ionization by the firstarriving pulse and avalanche ionization by the second arriving-pulse. Although the color center originated from the point defects (e.g. SiE' and $\mathrm{NBOHC}$ ) and the oxygendeficient centers (ODC(II)) was induced, we have also demonstrated that the nanograting can be applied to the integrated polarization sensitive imaging filter which can be expected to realize a new type of sensor capable of recording the optical properties of partially polarized light. Additionally, we have also confirmed the formation of the nanograting structure inside $\mathrm{GeO}_{2}$ glass in the case of low pulse energy $(<0.2 \mu \mathrm{J})$. Increasing in laser energy within the focus volume leads the generation of molecular oxygen. We anticipate that such unexpected light-matter interaction expands the possibilities for femtosecond-laser micromachining.

\section{Acknowledgments and Appendixes}

This work was financially supported by JSPS KAKENHI Grant Number 22350093. We would like to thank Prof. Kazuyuki Hirao from Kyoto University, Prof. Peter G. Kazansky from University of Southampton and Prof. Jianrong Qiu from South China University of Technology for their kind suggestions and discussions.

\section{References}

[1] R. R. Gattas and E. Mazur: Nat. Photonics, 2, (2008) 219.

[2] R. Birngruber, C. Puliafito, A. Gawande, W. -Z. Lin, R. Schoenlein and J. Fujimoto: IEEE J. Quant. Electron., 23, (1987) 1836.

[3] Y. Shimotsuma, P. G. Kazansky, J. Qiu and K. Hirao: Phys. Rev. Lett., 91, (2003) 247705.

[4] M. Shimizu, M. Sakakura, S. Kanehira, M. Nishi, Y. Shimotsuma, K. Hirao and K. Miura: Opt. Lett. 36, (2011) 2161

[5] E. Bricchi, B. G. Klappauf and P. G. Kazansky: Opt. Lett., 29, (2004) 119.

[6] L. Sudrie, M. Franko, B. Prade and A. Mysyrowicz: Opt. Commun., 171, (1999) 279.

[7] Y. Shimotsuma, M. Sakakura, P. G. Kazansky, M. Beresna, J. Qiu, K. Miura and K. Hirao: Adv. Mater., 22, (2010) 4039.

[8] V. R. Bhardwaj, E. Simova, P. P. Rajeev, C. Hnatovsky, R. S. Taylor, D. M. Rayner and P. B. Corkum: Phys. Rev. Lett., 96, (2006) 057404.

[9] S. Richter, M. Heinrich, S. Döring, A. Tünnermann, S. Nolte and U. Peschel: J. Laser Appl., 24, (2012) 042008.

[10] C. Hnatovsky, R. S. Taylor, P. P. Rajeev, E. Simova, V. R. Bhardwaj, D. M. Rayner and P. B. Corkum: Appl. Phys. Lett., 87, (2005) 014104.

[11] J. Canning, M. Lancry, K. Cook, A. Weickman, F. Brisset and B. Poumellec: Opt. Mater. Express, 1, (2011) 998.

[12] Y. Shimotsuma, M. Sakakura and K. Miura: Opt. Mater. Express, 1, (2011) 803.

[13] J. D. Mills, P. G. Kazansky, E. Bricchi and J. J. Baumberg: Appl. Phys. Lett., 81, (2002) 196.

[14] L. Bressel, D. de Ligny, E. G. Gamaly, A. V. Rode and S. Juodkazis: Opt. Mater. Express 1, (2011) 1150.

[15]L. Bressel, D. de Ligny, C. Sonneville, V. MartinezAndrieux and S. Juodkazis: J. Non-Cryst. Solids, 357, (2011) 2637.

[16] K. Sugioka, M. Iida, H. Takai and K. Micorikawa: Opt. Lett., 36, (2011) 2734.

[17]P. G. Kazansky, W. Yang, E. Bricchi, J. Bovatsek, A. Arai, Y. Shimotsuma, K. Miura and K. Hirao: Appl. Phys. Lett., 90, (2007) 151120.

[18]A. Mouskeftaras, S. Guizard, N. Fedorov and S. Klimentov: Appl. Phys. A, (2013) 110, 709.

[19] Y. Shimotsuma, T. Asai, K. Miura, K. Hirao and P. G. Kazansky: J. Laser Micro/Nanoengineering, 7 (2012) 339.

[20]L. Skuja: J. Non-Cryst. Solids, 239, (1998) 16.

[21] L. Skuja: J. Non-Cryst. Solids, 179, (1994) 51. 
[22] M. Lancry, B. Poumellec, R. Desmarchelier and B. Bourguignon: Opt. Mater. Express, 2, (2012) 1809.

[23] M. Shimizu, M. Sakakura, M. Ohnishi, Y. Shimotsuma, T. Nakaya, K. Miura and K. Hirao: J. Appl. Phys., 108, (2010) 073533.

[24] Y. Shimotsuma, K. Hirao, J. Qiu and K. Miura: J. Non-Cryst. Solids, 352, (2006) 646.
[25] M. Sakakura, M. Shimizu, Y. Shimotsuma, K. Miura and K. Hirao: Appl. Phys. Lett., 93, (2008) 231112.

[26] I. Daniel, P. Gillet, B. T. Poe and P. F. McMillan: Phys. Chem. Miner., 22, (1995) 74.

[27]D. J. Durben, G. H. Wolf: Phys. Rev. B, 43, (1991) 2355.

(Received: July 19, 2013, Accepted: December 28, 2013) 